\title{
Notas sobre la ecología reproductiva y conservación de los chor- los nevados Charadrius nivosus occidentalis en Paracas, Perú
}

\author{
Notes on the breeding ecology and conservation of snowy plovers \\ Charadrius nivosus occidentalis in Paracas, Peru
}

\author{
Clemens Küpper ${ }^{1}$, Edgardo Aguilar² y Oscar González ${ }^{3}$
}

\begin{abstract}
1 Museum of Comparative Zoology, Department of Organismic and Evolutionary Biology, Harvard University Cambridge, MA 02138 2 Grupo Aves del Perú-Pisco. Buenos Aires 318 San Andrés, Pisco. Ica, Perú. edgardoaguilarh@ yahoo.com

3 Grupo Aves del Perú. Gómez del Carpio 135, Barrio Medico, Lima 34. Peru. Dirección Actual: School of Natural Resources and the Environment, 103 Black Hall, University of Florida. Gainesville, Fl32611. USA. pajarologo@ufl.edu
\end{abstract} Aceptado: 21/02/201 Publicado online: $23 / 06 / 201$

\begin{abstract}
Resumen
El chorlo nevado es un ave playera endémica de las Americas, la cual tiene tres subespecies. En Sudamerica la subespecie occidentalis se encuentra a lo largo de la costa del océano Pacifico. Aunque se conoce su comportamiento reproductivo, demografía poblacional y éxito reproductivo en Norteamerica, poco se sabe de estos parámetros ecológicos de la subespecie occidentalis. En octubre de 2008 estudiamos la ecología reproductiva del chorlo nevado en la Reserva Nacional de Paracas, Ica, sudoeste del Perú. Los chorlos nevados fueron encontrados en todas las nueve playas arenosas y humedales costeros visitados. De acuerdo con los conteos estimamos que la población en Paracas consiste en por lo menos 500 chorlos nevados. Hubo evidencia de actividad reproductiva en seis de los nueve sitios. La mayoría de la actividad de crianza fue observada en Playón/Mendieta, alrededor de un charco salado temporal. Encontramos dos nidos y diez familias en toda la reserva. En total se capturaron y marcaron 24 polluelos, ocho machos y siete hembras. Las familias eran atendidas por ambos padres. Hasta el final del estudio 21 de los polluelos habían perecido. Durante el período de estudio ninguno de los polluelos marcados alcanzaron la edad de volantón de 25 días y solamente confirmamos que un polluelo en una visita posterior llego a volantón. Nuestras observaciones destacan algunos problemas observados durante el período reproductivo del chorlo nevado, también sugerimos acciones para contrarrestar las amenazas identificadas.
\end{abstract}

Palabras clave: Aves playeras, Reserva Nacional de Paracas, Charadrius nivosus (alexandrinus), ecología, reproducción, conservación.

\section{Abstract}

The snowy plover is a shorebird endemic to the Americas. It consists of three subspecies. In South America the subspecies occidentalis is found along the coast of the Pacific ocean. Although breeding behaviour, population demography and reproductive success are well established in North America, little is known about these ecological parameters for occidentalis snowy plovers. In October 2008 we studied breeding ecology of snowy plovers in the National Reserve Paracas, Ica, Peru. Snowy plovers were found at all nine sandy beaches and coastal wetlands visited. Based on counts we estimate the population in Paracas to consist of a minimum of 500 snowy plovers. Evidence for breeding activity was found at six of nine sites. Most breeding activity was observed at Playón/Mendieta, surrounding a temporal salt lagoon. Two nests and ten families were found in the entire reserve. In total 24 chicks, eight males and seven females were captured and marked. Families were tended by both parents. Twenty-one of the chicks had perished by the end of the study. During the intense study period none of the marked chicks reached the fledgling age of 25 days and only one chick was confirmed to have fledged during a subsequent visit. Our observations highlight threads during the snowy plover reproductive period that need to be addressed through conservation management and we suggest direct actions to counter the threads identified.

Keywords: Shorebirds, National Reserve Paracas, Charadrius nivosus (alexandrinus), ecology, reproduction, conservation.

\section{Introducción}

El chorlo nevado (snowy plover, Charadrius nivosus) es un ave playera pequeña con poblaciones residentes y migratorias en Norte y Sur America (del Hoyo et al. 1996, Hayman et al. 1986). Los chorlos nevados eran considerados como parte de los chorlos de Kent (Kentish plover) Charadrius alexandrinus hasta que un estudio reciente revelo profundas diferencias tanto genotípicas como fenotípicas entre los chorlos nevados de Eurasia y los americanos (Küpper et al. 2009).

Tres subespecies del chorlo nevado -nivosus, tenuirostris y occidentalis- son reconocidas actualmente (Hayman et al. 1986, del Hoyo et al. 1996, Funk et al. 2007, Küpper et al 2009). En Sudamérica, la subespecie occidentalis se distribuye a lo largo de las costas de Ecuador, Perú y Chile (Bullock 1936, González et al 1998, Pizarro-Solari 2004, Santander et al. 2006, Torres-Núñez 2007, comunicación personal de Ernesto Málaga). El plumaje es el criterio principal por el cual se define las subespecies: (1) los chorlos de la subespecie occidentalis tiene un antifaz más grande que las subespecies nivosus y tenuirostris además, (2) Los machos y hembras aparentan más similitud en el plumaje reproductivo que las otras dos subespecies americanas (Gorman 2001).

Los chorlos nevados y sus congéneres los chorlos de Kent tienen un sistema de reproducción muy flexible (Lessells 1984, Warriner et al. 1986, Székely \& Lessells 1993, Amat et al. 1999, Kosztolányi et al. 2009). Ambos padres incuban los huevos, pero cuando nace un polluelo usualmente uno de los padres abandona la nidada para aparearse nuevamente (generalmente la hembra). En una sola población reproductiva, puede haber poliandria, poliginia y monogamia. El cuidado de los polluelos puede ser realizado por uno o ambos padres. Los sistemas de reproducción pueden variar entre poblaciones y dentro de las poblaciones (Kosztolányi et al. 2009). Distintas variables como oportunidades de un nuevo apareamiento, presiones de depredación y disponibilidad de recursos han sido asociadas con esta variabilidad (Székely et al. 1999, Amat et al. 1999, Kosztolányi et al. 2006).

Los sitios de anidación de los chorlos nevados se pueden encontrar en playas arenosas y alrededor de charcos salados 
temporales en zonas templadas y subtropicales. Esta preferencia de hábitat se ha convertido en una problemática para la especie debido a que playas arenosas no perturbadas son muy raras por su uso en actividades de recreación humana u otras actividades económicas. En las últimas décadas las poblaciones de esta ave están declinando en Norteamérica y Europa (Stroud et al. 2004, Page et al. 2009). La perturbación por actividades de recreación en las áreas reproductivas influencio en los fracasos reproductivos de chorlos playeros observados en playas de California (Ruhlen et al. 2003). Esta declinación ha llevado a que los chorlos nevados estén bajo protección estricta en Norteamérica, donde las poblaciones del Pacifico están protegidas bajo el Acta de especies amenazadas (US Endangered Species Act, USFWS 1993). Sin embargo, poco se sabe de la abundancia, tendencias poblacionales y comportamiento de los chorlos sudamericanos, los cuales son genéticamente diferentes de otros chorlos nevados (Funk et al. 2007).

En el presente trabajo nosotros estudiamos la ecología reproductiva de los chorlos nevados en la Reserva Nacional de Paracas (RNP) en Ica, Perú. La RNP es parte del desierto costero pacifico entre Perú y Chile; uno de los sitios más secos del mundo (Brack y Mendiola 2000). Las temperaturas son moderadas con poca variación durante el año (promedio $18,9^{\circ} \mathrm{C}$ ) y la precipitación anual es menor de $2 \mathrm{~mm}$ (Craig and Psuty 1968). Se observa una importante interacción entre el ecosistema marino de la corriente de Humboldt y los organismos costeros (Catenazzi \& Donelly 2007). Muchas aves playeras usan la reserva como un sitio de invernada (Duffy et al. 1981); de estas aves, el chorlo nevado es una de las pocas especies que se reproduce en Paracas.

Este estudio tuvo como objetivos (1) identificar los sitios de reproducción de los chorlos nevados (2) contar los chorlos nevados para estimar su tamańo poblacional, (3) estudiar los movimientos de adultos y juveniles mediante el marcado de individuos (4) investigar el éxito reproductivo y el comportamiento de cuidado parental de las familias de chorlos y (5) identificar amenazas para la población reproductiva.

\section{Material y métodos}

El trabajo de campo en la Reserva Nacional de Paracas - RNP $\left(13^{\circ} 52 \mathrm{~S}, 76^{\circ} 16 \mathrm{~W}\right)$ fue del 03 al 25 de octubre de 2008 . Los sitios potenciales de anidación fueron identificados (1) utilizando imágenes satelitales disponibles en Google Earth ubicando humedales y playas adecuadas (Fig.1), (2) por información proporcionada por guardaparques de la RNP, (3) por nuestras observaciones previas en la RNP. En cada lugar contamos el numero de chorlos y registramos signos de actividad reproductiva como machos cortejando, nidos vacios, nidos con huevos o familias, chorlos cuidando polluelos durante observaciones breves. Usamos los métodos de campo de Székely et al. (2008), adaptados a las condiciones locales. Las observaciones usualmente se llevaron a cabo en las primeras horas de la mañana $(6-11 \mathrm{am})$ y en la tarde $(5-7 \mathrm{pm})$ cuando había poco viento. Buscamos áreas con nidos caminando y ahuyentando chorlos que incubaban, buscando en el suelo por nidos o identificando chorlos incubando desde un carro o escondites móviles. Cuando un nido fue encontrado medimos el largo de los huevos usando un escalímetro al 0,1 $\mathrm{mm}$, determinamos la edad de la nidada haciendo flotar los huevos en agua y tomamos la posición geográfica de los nidos. Los nidos se marcaron con una señal aproximadamente a 15 metros de distancia.
Los chorlos adultos se capturaron utilizando dos métodos, con redes de neblina y con trampa de embudo. Con el primer método, las redes de neblina de aproximadamente $50 \mathrm{~m}$ se pusieron en cuatro sitios donde encontramos chorlos en abundancia. Los chorlos fueron capturados en la noche o en mañanas nubladas. El segundo método, consistió en colocar trampas de embudo en (1) el nido, (2) usando pichones un atrayente para capturar adultos en reproducción. Cada adulto se marcaba con una combinación única de un anillo de metal y tres anillos de color para identificarlos luego en el campo. Medimos el tarso (hasta $0,1 \mathrm{~mm}$ ), longitud de ala (hasta el milimetro, usando el método de la máxima cuerda alar), masa corporal (hasta $0,5 \mathrm{~g}$ ).

Los polluelos fueron anillados luego de nacer en el nido o cuando eran cuidados por los padres. Cada polluelo fue marcado con un anillo de metal y un anillo de color; les medimos el tarso $( \pm 0,1 \mathrm{~mm})$ y la masa corporal $( \pm 0,1 \mathrm{~g})$.

Buscamos las áreas que podrían ser adecuadas para el anidamiento de familias y chorlos marcados. En cada encuentro anotamos la combinación de color y el sexo del chorlo marcado, y la posición geográfica utilizando un GPS con la posición del observador y estimamos la distancia en la que se encontraba el chorlo. Cuando se volvían a avistar las familias, también anotamos el número de los polluelos y las combinaciones de color de los anillos de todos los miembros de la familia presentes.

\section{Resultados}

Censos de chorlos nevados en reproducción.- Visitamos nueve sitios que aparentaron ser adecuados para la reproducción de los chorlos nevados. En seis de los nueve sitios encontramos evidencia de actividad reproductiva (Tabla 1, Fig. 1).

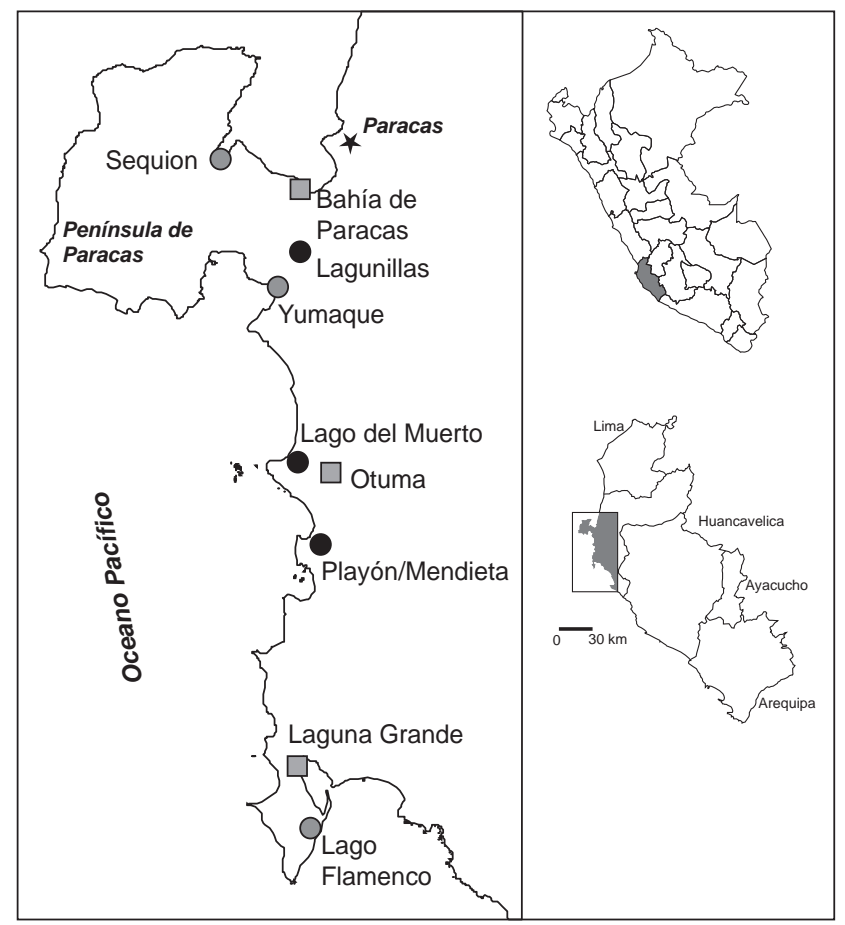

Figura 1. Mapa de los áreas de conteo y reproducción del chorlito nevado Charadrius nivosus occidentalis en la Reserva Nacional de Paracas, en octubre 2008. Los cuadrados representan lugares donde se observo al chorlo nevado pero no se hallo signos de reproduccion. Los círculos grises representan sitios donde hubo comportamiento reproductivo (machos en cortejo, nidos vacios) pero no se hallaron huevos o polluelos. Los círculos negros representan sitios donde encontramos huevos y/o polluelos. 
Tabla 1. Sitios de importancia para la reproducción de chorlos nevados Charadrius nivosus occidentalis en la Reserva Nacional de Paracas.

\begin{tabular}{|c|c|c|c|c|}
\hline Sitio & Localización geográfica & Fecha & $\begin{array}{c}\text { Número de chorlos } \\
\text { nevados }\end{array}$ & Evidencia de actividad reproductiva \\
\hline Sequión & $\mathrm{S} 13^{\circ} 50^{\prime} 52, \mathrm{~W} 76^{\circ} 18^{\prime} 35$ & 07 Octubre & 37 & $\begin{array}{l}\text { - } 5 \text { machos en display } \\
\text { - Varios nidos vacios } \\
\text { - } 3 \text { parejas copulando }\end{array}$ \\
\hline Lago Flamenco & $\mathrm{S} 14^{\circ} 10^{\prime} 24, \mathrm{~W} 76^{\circ} 15^{\prime} 45$ & 08 Octubre & 9 & $\begin{array}{l}\text { - } 1 \text { macho en display } \\
\text { - } 2 \text { nidos vacios }\end{array}$ \\
\hline Laguna Grande & $\mathrm{S} 14^{\circ} 08^{\prime} 28, \mathrm{~W} 76^{\circ} 16^{\prime} 12$ & 08 Octubre & 45 & - ninguno \\
\hline Lago del Muerto & $\mathrm{S} 13^{\circ} 59^{\prime} 37, \mathrm{~W} 76^{\circ} 16^{\prime} 14$ & 04 Octubre & 65 & $\begin{array}{l}\text { - } \quad>10 \text { machos en display } \\
\text { - } 3 \text { nidos vacios } \\
\text { - } 1 \text { familia con un pichón }\end{array}$ \\
\hline Lagunillas & $\mathrm{S} 13^{\circ} 53^{\prime} 33, \mathrm{~W} 76^{\circ} 18^{\prime} 51$ & 04 Octubre & 28 & $\begin{array}{l}\text { - } 3 \text { machos en display } \\
\text { - } 1 \text { familia con dos pichones }\end{array}$ \\
\hline Otuma & S $13^{\circ} 59^{\circ} 59, \mathrm{~W} 76^{\circ} 15^{\prime} 16$ & 24 Octubre & 115 & - ninguna \\
\hline Bahía de Paracas & $\mathrm{S} 13^{\circ} 51^{\prime} 45, \mathrm{~W} 76^{\circ} 16^{\prime} 09$ & 25 Octubre & 28 & - ninguna \\
\hline Playón/Mendieta & $\mathrm{S} 14^{\circ} 01^{\prime} 59, \mathrm{~W} 76^{\circ} 15^{\prime} 39$ & 09 Octubre & $72(150)^{*}$ & $\begin{array}{l}\text { - }>10 \text { machos en display } \\
\text { - } 80 \text { nidos vacios } \\
\text { - } 2 \text { nidos } \\
\text { - } 8 \text { familias }\end{array}$ \\
\hline Yumaque & $\mathrm{S} 13^{\circ} 54^{\prime} 39, \mathrm{~W} 76^{\circ} 16^{\prime} 55$ & 05 Octubre & 6 & - 2 nidos vacios \\
\hline
\end{tabular}

*Puntos de conteo a lo largo de transecto a través del sitio de reproducción. Los conteos cubrieron aproximadamente el 50\% del área total del sitio. La cantidad total estimada se da en paréntesis.

El sitio con la mayor actividad reproductiva y el mayor número de chorlos estimado fue Playón/Mendieta. Los chorlos en cortejo se encontraron en barrizales extensos y campos pedregosos alrededor de una laguna temporal que fue creada por inundación del mar después del terremoto de 2007. Enfocamos nuestros estudios en la ecología reproductiva y el éxito reproductivo en esta área.

Capturas.- Capturamos en total 39 chorlos nevados (siete hembras, ocho machos y 24 juveniles). Tres chorlos adultos fueron capturados en el nido mientras incubaban, cuatro chorlos adultos fueron capturados usando sus crías como carnada y ocho chorlos adultos fueron capturados con redes de neblina. Todos los pichones excepto dos que fueron anillados en el mismo nido fueron capturados en el territorio reproductivo. Las principales medidas corporales se dan en la Tabla 2.

Ecología reproductiva.- Los machos y hembras de los chorlos nevados durante el periodo reproductivo tienen ornamentos negros en la cabeza, pecho y ojos. Los chorlos adultos de ambos sexos estuvieron ornamentados durante el periodo de estudio en Paracas. Esto sugiere que la estación reproductiva ya había empezado cuando inició el estudio. También frecuentemente encontramos parejas de chorlos nevados comiendo juntos. A pesar de una búsqueda intensiva solo encontramos dos nidos con huevos, ambos en Playón/Mendieta. En uno de los nidos los huevos eclosionaron, mientras que el otro fue abandonado por los padres días antes de la eclosión. Contrasta los pocos nidos activos $(n=2)$ que encontramos con más de 80 cubetas de nidos vacios.

Marcamos pichones de diez familias. Polluelos y adultos de ocho familias fueron marcadas en Playón/Mendieta, de una familia en Lagunillas y de una familia en Lago del Muerto. Con excepción de dos familias, todas estaban compuestas de ambos padres. No se pudo determinar el sexo de los padres solitarios que cuidaban polluelos. Pocas familias volvieron a ser avistadas.

Observamos diferencias en los patrones de cuidado de pichones de los chorlos nevados de Paracas en comparación con otras poblaciones de chorlos; todas las familias de Paracas fueron biparentales con uno de los padres usualmente cerca cuidando los polluelos mientras que el otro estaba alimentándose a cierta distancia ( $n=16$ observaciones). El padre alerta mostro un com-

Tabla 2. Características morfológicas de chorlos nevados Charadrius nivosus occidentalis capturados, adultos y juveniles, en Paracas. Se muestra la media y la desviación estándar.

\begin{tabular}{lccc}
\hline & Longitud de tarso [mm] & Masa corporal [g] & Longitud de ala [mm] \\
\hline Adultos & & & \\
Machos $(\mathbf{n}=\mathbf{8})$ & $25,7 \pm 0,97$ & $38,0 \pm 2,88$ & $109 \pm 2,77$ \\
Hembras $(\mathbf{n}=7)$ & $25,0 \pm 0,65$ & $40,8 \pm 3,45$ & $110 \pm 2,03$ \\
Juveniles $(\mathbf{n}=\mathbf{2 4})$ & & & \\
- Encontrados en el nido $\quad 18,8 \pm 0,11$ & $7,3 \pm 0,14$ & No medido \\
- Encontrados luego que la familia dejo el nido & $20,1 \pm 0,78$ & $8,2 \pm 3,15$ & No medido \\
\hline
\end{tabular}


portamiento de defensa territorial mientras que el padre distante aparentaba no ser territorial. El padre que no estaba en guardia solo venia a asistir en defensa de la familia cuando los polluelos eran atacados por otros chorlos nevados adultos o perturbados por los humanos o predadores potenciales ( $\mathrm{n}=9$ observaciones). En tres de las cuatro familias que fueron encontradas más de una vez, distintos padres estaban cuidando los polluelos durante las diferentes observaciones.

Hasta el final del estudio no observamos ningún pichón que se independice. Tres de 24 pichones anillados (12,5\%) se reavistaron al final del periodo de estudio. Estos pichones fueron de dos familias, una en Playón/Mendieta y otra en Lagunillas. Los machos de otras dos familias fueron reavistados solos en el área pocos días luego de haber sido vistos con sus pichones. Al reavistar ambos machos no estaban cuidando pichones y no mostraron ningún comportamiento defensivo cuando nos aproximamos, por lo que asumimos que los polluelos habían muerto (Szekely et al. 1999). Aparte de los chorlos que fueron capturados mientras anidaban solo un chorlo fue reavistado a pesar de revisar a más de 200 chorlos después de terminar la captura. Este chorlo, un macho, fue reavistado en Lagunillas a $250 \mathrm{~m}$ cerca del sitio de su captura luego de cuatro horas de haber sido soltado.

Amenazas.- El éxito reproductivo observado fue bajo. Los depredadores potenciales que se observaron en las áreas de anidación fueron gaviotas, halcones y zorros. La perturbación humana fue un problema, en Playón/Mendieta frecuentemente vehículos eran conducidos por la playa atravesando las áreas de reproducción, amenazando destruir los nidos y matar a los polluelos. También fueron observados motociclistas manejando por las áreas de reproducción y espantando a las aves playeras que descansaban.

Durante el periodo de estudio las playas fueron usadas por extractores para secar algas, las cuales luego son vendidas como insumos de fertilizantes o productos farmacéuticos. El impacto de esta actividad sobre la reproducción del chorlo nevado podría ser evaluado en próximos estudios. Por otro lado las algas extraídas atrajeron muchos insectos los cuales podrían ser alimento para las familias de chorlos y las aves que descansan, sin embargo, no observamos a ningún chorlo usando este recurso alimenticio.

\section{Discusión}

Nuestro estudio marca la importancia supraregional de la Reserva Nacional de Paracas para la subespecie occidentalis del chorlo nevado. Nuestros resultados sugieren que en la RNP se asienta una importante población de chorlos nevados. Se conoce muy poco sobre la reproducción y la abundancia de los chorlos nevados en Sudamerica; los encuentros con chorlos anidando son raros (Bullock et al 1936, Vilina et al 2009). Los chorlos nevados de la subespecie nivosus en el hemisferio norte han sido mejor estudiados y su población se estima en 18000 individuos en los EE.UU y México (Page et al. 2009); aunque muchos de los chorlos nevados norteamericanos anidan tierra adentro.

A diferencia de otras especies de aves costeras de Paracas, los chorlos nevados no solo usan las playas, sino que también pueden ser encontrados en barrizales alejados y cuerpos de agua internos en toda la RNP y donde son probablemente inadvertidos. En el presente trabajo encontramos chorlos en todas las playas arenosas, lagunas y cuerpos de agua internos que visitamos.
Basados en los censos y reavistamientos de chorlos marcados con anillos de colores, consideramos que la población total de chorlos nevados tiene un mínimo de 500 individuos en toda la RNP. También observamos dos chorlos adultos anillados en la misma zona donde fueron capturados después de dos años, en área de Lagunillas.

El plumaje y las medidas estructurales del cuerpo tales como la longitud del tarso y de las alas del chorlo nevado en paracas fueron muy similares a los de la subespecie nivosus que anida en el noroeste de México (Küpper et al. 2009). Esto apoya el punto de vista que las dos subespecies pueden estar poco diferenciadas, lo cual también se encontró cuando se analizaron marcadores genéticos neutrales (Funk et al. 2007). Sin embargo, la ecología reproductiva fue muy diferente. Primero, mientras los chorlos nevados tienen un periodo reproductivo muy intenso, la actividad reproductiva en la RNP fue muy pobre. Muchas hembras de nivosus produjeron varias nidadas (hasta 4) por estación y muchos chorlos estuvieron anidando en ese tiempo (Küpper et al. 2007a \& datos no publicados). A pesar que observamos muchos machos y hembras en parejas solo una pequeña fracción se estaba reproduciendo en durante nuestras observaciones en Paracas.

Segundo, los chorlos nevados en el noroeste de México son comúnmente poliandricos con la hembra abandonando la familia pocos días luego de la eclosión los huevos y los machos inusualmente proveen el cuidado de los polluelos solos. En Paracas se encontró que fue predominante el cuidado biparental. Estas diferencias pueden ser causadas por la calidad del ambiente de reproducción. El ambiente reproductivo en el noroeste de México parece ser mas rico en alimento mientras que los recursos alimenticios son escasos en el desierto costero de Paracas (Pero ver Erckmann 1983). Sin embargo, nuestros datos sólo se refieren a una parte del periodo reproductivo de los chorlos nevados de Paracas, porque es muy probable que la estación reproductiva sea más larga. El cuidado parental varía entre las estaciones en los chorlos nevados y de Kent; por lo que el comportamiento de cuidado parental puede variar luego de la estación reproductiva (Kosztolányi et al. 2006, Küpper et al. 2007a).

El bajo éxito reproductivo observado en la RNP es una amenaza importante para los chorlos nevados. La actividad de reproducción fue pobre y solo 3 de los 24 polluelos marcados fueron observados vivos al final del estudio. Dos semanas luego del estudio uno de los 3 polluelos sobrevivientes fue encontrado muerto en Lagunillas, mientras que solo reavistamos un polluelo que alcanzo más de 25 días. Las causas de esta alta mortalidad deben ser reconocidas como un punto crítico en el manejo de la población del chorlo nevado.

\section{Recomendaciones}

Investigación.- Luego de establecer que un gran número de chorlos nevados usan la Reserva Nacional de Paracas, se debe realizar un estudio más intensivo del éxito reproductivo del chorlo nevado en ese lugar. Este estudio debe determinar la duración de la estación reproductiva, estudiar el éxito reproductivo y el comportamiento de los chorlos nevados en más detalle.

Se necesita establecer si la actividad reproductiva y el éxito reproductivo son mayores en distintas ocasiones del año y entre diferentes ańos. Los chorlos nevados son aves longevas que pueden vivir aproximadamente hasta 15 años (Page et al. 2009). Debido a las difíciles condiciones ambientales y la variabilidad 
interanual el éxito reproductivo y actividad reproductiva en la RNP puede cambiar entre los años y algunos chorlos podrían criar solamente cuando las condiciones sean favorables, por lo que se necesita un estudio a largo plazo para conseguir suficiente información para un plan de conservación efectivo para la población.

Los chorlos nevados se pueden encontrar en las orillas marinas de la RNP todo el año. Sin embargo, no está claro si las aves observadas eran residentes y las mismas aves permanecen en los sitios cada ańo o si ellas se están moviendo a lo largo de la costa peruana y diferentes chorlos nevados están utilizando la RNP para pasar el invierno y reproducirse. Entonces el marcaje con colores de los chorlos y su monitoreo permitirían entender sus movimientos, así como estimar el tamaño poblacional.

Las diferencias de comportamiento, las cuales son muy marcadas entre distintas poblaciones de chorlos nevados merecen especial atención. Un estudio comparativo de poblaciones y la manipulación experimental podrían probar la hipótesis de que la disponibilidad del recurso afecta la ecología reproductiva de los chorlos.

La conservación de la población del chorlo nevado en la RNP implica un estudio del flujo genético entre distintas poblaciones del Perú, los resultados ayudarían a la conservación supraregional de esta subespecie y podrá ser usado para la revisión de planes de acción para otras especies.

Acciones de Conservación.- La alta perturbación de las playas causada por humanos, probablemente ha causado un exodo de las familias de chorlos y puede forzarlos al uso de territorios para anidación menos perturbados; pero también menos favorables en recursos alimenticios. Recomendamos que el uso de la playa durante la estación reproductiva sea severamente limitado. El tránsito de vehículos 4X4, motos y bicicletas deberían ser regulado para evitar la perturbación de las nidadas y mortalidad de chorlos. La regulación y el uso de caminos bien señalizados debe ser en en especial en sitios de importancia reproductiva para el chorlo: Lagunillas, Lago Muerto, Playón/Mendieta y Sequión.

Las señales en los sitios de reproducción deben ser usadas para sensibilizar la conciencia ambiental de la gente local y los turistas. El Lago del Muerto y el Sequión albergaron un gran número de aves migratorias y parecen ser buenos sitios para ecoturismo.

En nuestro estudio Playón/Mendieta fue el sitio más importante para anidar en la RNP. Sin embargo, algunos indicadores señalan que el uso del lugar para actividad reproductiva fue solamente ocacional y causada por una inundación temporal. Hacia el final de nuestro estudio el agua se evaporo completamente y queda por investigar si los chorlos aun usan este sitio para reproducción. Otros sitios adecuados para anidar que pueden albergar a una sustancial población nidificante son los sitios de extracción de sal (salinas). Los sitios de extracción de sal a menudo proveen un hábitat adecuado para los chorlos (C Küpper, datos no publicados). Las Salinas de Otuma, un sitio activo de extracción de sal se localizan a pocos kilómetros al norte de Playón/Mendieta y se necesita establecer su importancia para los chorlos nidificantes.

Las algas varadas en las playas son una parte importante de la red trófica del desierto costero (Catenazzi \& Donelly 2007). Modificaciones en las biomasas de esas algas varadas podrían tener consecuencias directas sobre la biodiversidad terrestre de la RNP. Así mismo el incremento de la actividad extractiva y de las áreas de secado de las algas podría tener repercusiones sobre las zonas usadas por los chorlos. Recomendamos como muy importante la realización de un estudio de impacto ambiental de la extracción de algas a pequeńa y gran escala antes que se permitan planes de extracción de algas más extensivos dentro de la RNP.

\section{Agradecimientos}

Agradecemos al Profesor Tamás Székely, Universidad de Bath, Inglaterra, por su consejo y apoyo durante este estudio. Scott Robinson y su equipo de la Universidad de Florida proveyeron comentarios adicionales para mejorar este manuscrito. Mario Mighty de la Universidad de Florida ayudo en el diseño del mapa. Ernesto Málaga proveyó información valiosa del chorlo nevado anidando en el sur del Perú. El permiso de investigación fue otorgado por INRENA (042C/C-2008-INRENA-IANP). El estudio fue apoyado financieramente por National Geographic e INCORE.

\section{Literatura citada}

Amat J.A., R.M. Fraga \& G.M. Arroyo. 1999. Brood desertion and polygamous breeding in the Kentish Plover Charadrius alexandrinus. Ibis 141: 596-607.

Brack A. \& C. Mendiola. 2000. Ecologia. Editorial Bruño. Lima.

Bullock D. S. 1936. The snowy plover (Charadrius alexandrinus occidentalis) nesting in Chile. The Auk,53: 170-172.

Catenazzi A. \& M.A. Donelly. 2007. The Ulva connection: marine algae subsidize terrestrial predators in coastal Peru. OIKOS 116: 75-86.

Craig A.R. \& N.P. Psuty. 1968. The Paracas papers. Studies in marine desert ecology. In: Reconnaissance Report, pp. 1-196: Dept of Geography, Florida Atlantic Univ.

del Hoyo J., A. Elliot \& J. Sargatal. 1996. Handbook of the birds of the world. Hoatzin to Auks. Barcelona: Lynx edicions

Duffy D.C., N. Atkins \& D.C. Schneider. 1981. Do shorebirds compete on their wintering grounds? The Auk 98: 215-229.

Erckmann W.J. 1983. The evolution of polyandry in shorebirds: an evaluation of hypotheses. In: Social behaviour of female vertebrates (Ed. Wasser, S. K.), pp: 113-168. New York: Academic Press.

Funk W.C., T.D. Mullins \& S.M. Haig. 2007. Conservation genetics of snowy plovers (Charadrius alexandrinus) in the Western Hemisphere: population genetic structure and delineation of subspecies. Conservation Genetics 8: 1287-1309.

González O., L. Pautrat \& J. González. 1998. Las aves mas comunes de Lima y alrededores. Editorial Santillana. Lima.

Gorman L.R. 2000. Population differentiation among snowy plovers (Charadrius alexandrinus) in North America. Master of Science in Wildlife Science. Oregon State University.

Hayman P., J. Marchant \& T. Prater. 1986. Shorebirds: an identification guide to the waders of the world Boston: Houghton Mifflin Co.

Kosztolányi A., T. Székely, I.C. Cuthill, K.T. Yilmaz \& S. Berberoğlu. 2006. Ecological constraints on breeding system evolution: the influence of habitat on brood desertion in Kentish plover. Journal of Animal Ecology 75: 257-265.

Kosztolányi A., S. Javed, C. Küpper, et al. 2009. Breeding ecology of Kentish Plover Charadrius alexandrinus in an extremely hot environment. Bird Study, 56, 244-252.

Küpper C., L. Lozano-Angulo, M. Cruz-Lopez, \& M. Bucio Pacheco. 2007a. Reproductive success and parental care of snowy plover Charadrius alexandrinus nivosus in Ceuta, Mexico. Reporte no publicado. University of Bath. 
Küpper C., G.J. Horsburgh, D.A. Dawson, et al. 2007b. Characterization of 36 polymorphic microsatellite loci in the Kentish plover (Charadrius alexandrinus) including two sex-linked loci and their amplification in four other Charadrius species. Molecular Ecology Notes 7: 35-39.

Küpper C., J. Augustin, A. Kosztolányi, et al. 2009. Kentish versus Snowy Plover: Phenotypic and genetic analyses of Charadrius alexandrinus reveal divergence of Eurasian and American subspecies. Auk 126: 839-852.

Lessells C.M. 1984. The mating system of Kentish plovers Charadrius alexandrinus. Ibis 126: 474-483.

Page G. W., L.E. Stenzel, G.W. Page, et al. 2009. Snowy Plover (Charadrius alexandrinus). The Birds of North America Online (A. Poole, Ed.). Ithaca: Cornell Lab of Ornithology; Retrieved from the Birds of North America Online: http://bna.birds.cornell.edu/bna/species/154. (acceso: 24/11/2010)

Pizarro-Solari C. A. 2004. Áreas marinas protegidas y su utilidad en la conservación de las aves marinas en Chile. Tesis para Titulo de Biólogo. Universidad de Chile, p: 114. http:// www.cybertesis.cl/tesis/uchile/2004/pizarro_c/sources/ pizarro_c.pdf (acceso: 24/11/2010)

Ruhlen T.D., S. Abbott, L.E. Stenzel \& G.W. Page. 2003. Evidence that human disturbance reduces snowy plover chick survival. Journal of Field Ornithology 74: 300-304.

Santander T., J.R. Hidalgo \& B. Haase. 2006. Reporte final. Aves acuáticas en Ecuador. Waterbird conservation for the Americas. Aves \& Conservacion Corporacion Ornitologica del Ecuador, Quito, p 53.
Stroud D.A., N.C. Davidson, R.West, D.A. Scott, et al. 2004. Status of migratory wader populations in Africa and western Eurasia in the 1990s. International Wader Studies 15.

Székely T. \& C.M. Lessells. 1993. Mate change by Kentish Plovers Charadrius alexandrinus. Ornis Scandinavica 24: 317-322.

Székely T., I.C. Cuthill \& J. Kis. 1999. Brood desertion in Kentish plover: sex differences in remating opportunities. Behavioral Ecology 10: 185-190.

Székely T., A. Kosztolanyi \& C. Küpper. 2008. Practical guide for investigating breeding ecology of Kentish plover Charadrius alexandrinus. University of Bath.

Torres-Nuñez M. 2007. Evaluación Ornitológica de los Humedales de Puerto Viejo, Pantanos de Villa y Humedales de Ventanilla. En: Serie de publicaciones de flora y fauna silvestre. Lima: Instituto Nacional de Recursos Naturales, Lima Perú. <http://issuu.com/leonardo.editor/docs/publicaciones_del_inrena> (acceso: 16/06/2011 -el editor)

USFWS (US Fish and Wildlife Service). 1993. Determination of threatened status for the Pacific Coast population of the western snowy plover. 58:12864-12874. Federal Register.

Vilina Y.A., P.A. Sáez, H. Cofré \& C.F. Garín. 2009. Nidificación del chorlo nevado (Charadrius alexandrinus) en las planicies costeras del desierto de Atacama, Chile. Boletín Chileno de Ornitología 15: 42-47.

Warriner J.S., J.C. Warriner, G.W. Page \& L.E. Stenzel. 1986. Mating system and reproductive success of a small population of polygamous snowy plovers. Wilson Bulletin 98: 15-37. 